\title{
A possible mechanism to tune magneto-electroluminescence in organic light-emitting diodes through adjusting the triplet exciton density
}

\author{
P. Chen, ${ }^{1,2}$ Q. L. Song, ${ }^{1}$ W. C. H. Choy, ${ }^{2}$ B. F. Ding, ${ }^{2}$ Y. L. Liu, ${ }^{1}$ and Z. H. Xiong ${ }^{1, a)}$ \\ ${ }^{1}$ School of Physical Science and Technology, MOE Key Laboratory on Luminescence and Real-Time Analysis, \\ Southwest University, Chongqing 400715, People's Republic of China \\ ${ }^{2}$ Department of Electrical and Electric Engineering, The University of Hong Kong, Pokfulam Road, \\ Hong Kong
}

(Received 1 June 2011; accepted 3 September 2011; published online 4 October 2011)

\begin{abstract}
At $15 \mathrm{~K}$, the high field decay of magneto-electroluminescence (MEL) in tris (8-hydroxyquinoine) aluminum $\left(\mathrm{Alq}_{3}\right)$-based organic light-emitting diodes is greatly suppressed by mixing hole transport material N, $\mathrm{N}^{\prime}$-bis(naphthalen-1-y)-N, $\mathrm{N}^{\prime}$-bis(phenyl) benzidine (NPB) into $\mathrm{Alq}_{3}$ emission layer. The positive correlation between the MEL decay and the triplet exciton density indicates the reduced triplet exciton density is the major contributing factor. The change of triplet exciton density and then the MEL upon NPB mixing may result from the strengths change of charge-triplets interaction, in which the injected charges flowing through the exciton formation zone without forming static space charges play the most important role. (C) 2011 American Institute of Physics.

[doi:10.1063/1.3644147]
\end{abstract}

Because of great potential in revealing the nature of the generated excitonic states and their relaxation dynamics in organic optoelectronic semiconductors, the magnetic field effects on electroluminescence (EL) in organic light-emitting diodes (OLEDs) called magneto-electroluminescence (MEL) has been attracted intense investigation. ${ }^{1-13}$ The MEL is defined as $\mathrm{MEL}=\Delta \mathrm{EL} / \mathrm{EL}=[E L(B)-E L(0)] / E L(0)$, where $E L(B)$ and $E L(0)$ are the EL intensity with and without external magnetic field $(B)$, respectively. Although many efforts have been devoted to studying the MEL, it is still unclear which factors govern the feature of MEL and, hence, the method of MEL controlling is under development.

The organic magnetic field effects may be caused by several $B$-dependent physical processes in organic semiconductor devices, including the generations of bipolarons, ${ }^{1,14}$ the spin-mixing between singlet and triplet through hyperfine interaction, ${ }^{2-4}$ the quenching of triplets through tripletscharge interaction ${ }^{3,4,15}$ or through triplets-cathode reaction ${ }^{3}$ or through the triplet-triplet annihilation (TTA) process, ${ }^{4,7-9,11}$ the $B$-induced mobility change of carriers, ${ }^{5,6}$ and even the isotope effect in spin response. ${ }^{10} \mathrm{We}$ have recently discovered a significant, high fields $(>45 \mathrm{mT})$ decay of MEL in pristine tris (8-hydroxyquinoine) aluminum $\left(\mathrm{Alq}_{3}\right)$-based OLEDs at low temperature below $100 \mathrm{~K}^{9}$ and in fluorescent dye doped $\mathrm{Alq}_{3}$-based OLEDs even at room temperature, ${ }^{11}$ indicating the magnetic field dependent TTA process is one possible mechanism for the MEL decay. In this mechanism, the behavior of the MEL at high field region is determined by the strength of TTA process which principally depends on the triplet exciton density: the higher triplet exciton density is, the stronger TTA process occurs and consequently more remarkable high field decay of MEL can be seen. ${ }^{4,7-9,11}$ However, there is no direct theoretical and experimental evidence for the relationship between the MEL and the triplets in the OLEDs. If continuous MEL change can be achieved by controlling the triplet exciton density in

a)Electronic mail: zhxiong@swu.edu.cn.
OLEDs, such as controlling their formation in the exciton formation zone, this relationship can be verified and can be used to tune the MEL in organic semiconductors.

In this letter, the MEL was studied by mixing the conventional hole transport material of $\mathrm{N}, \mathrm{N}^{\prime}$-bis(naphthalen1-y)-N,N'-bis(phenyl)benzidine (NPB) into $\mathrm{Alq}_{3}$ emission layer (EML). This structure allows the injected charge carriers flowing through the exciton formation zone to interact with triplets without forming the space charges accumulating at the abrupt $\mathrm{NPB} / \mathrm{Alq}_{3}$ interface. We found the MEL decay at $15 \mathrm{~K}$, unlike the remarkable decay trend of MEL in unmixed device (OLEDs with pristine $\mathrm{Alq}_{3}$ EML), is greatly suppressed in the NPB-mixed device. The rate model shows the MEL decay and triplet exciton density consistently vary with NPB concentration in the mixed EML, suggesting a possible mechanism to tune the MEL through adjusting the triplet exciton density. The capacitance-voltage $(\mathrm{C}-\mathrm{V})$ characteristics of NPB-mixed devices give the further evidence for the release of static space charges.

The OLEDs with structure of indium tin oxide (ITO)/ NPB $(60 \mathrm{~nm}) / \mathrm{Alq}_{3}: \mathrm{NPB}$ (weight ratio $\approx 0 \%-98 \%, 50 \mathrm{~nm}$ )/ $\mathrm{Alq}_{3}(30 \mathrm{~nm}) /$ lithium fluoride $(0.7 \mathrm{~nm}) / \mathrm{Al}$ were fabricated with active area of $1 \times 2 \mathrm{~mm}^{2}$. The sample preparation and the MEL measurement were described previously. ${ }^{4,7-9,11}$ The C-V characteristics were obtained using the Agilent 4294A Impedance Analyzer at the frequency of $1 \mathrm{kHz}$ in the ambient environment at room temperature.

Figs. 1(a) and 1(b), respectively, show the injection current dependence of $\triangle E L / E L$ without and with the NPBmixed EML at $15 \mathrm{~K}$. The MEL is composed of two effects in different regimes: a low field effect $(0<\mathrm{B}<45 \mathrm{mT})$ and a high field effect $(>45 \mathrm{mT})$. For the unmixed device, the high field part shows a remarkable decay after a rapid rising within low field range. However, the curves of MEL change greatly when the EML is mixed with 50 wt. \% NPB: (1) although the low field increase is still present, the value becomes larger; (2) the high field decay almost vanishes. What is more, we can also see the difference of temperature 


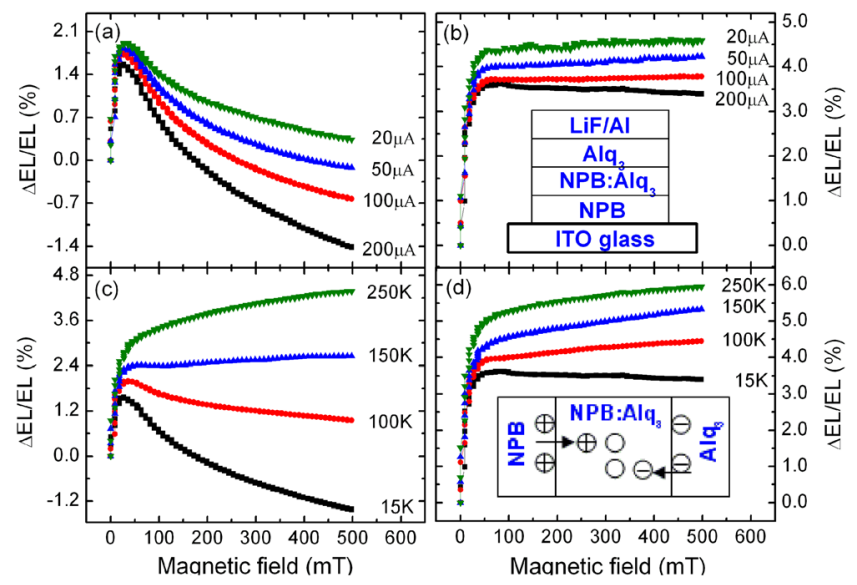

FIG. 1. (Color online) $\triangle \mathrm{EL} / \mathrm{EL}$ versus magnetic fields (MEL) for devices without and with 50\% NPB-mixed EML [(a) and (b)] with the same injection current of $200 \mu \mathrm{A}$ at different temperatures and [(c) and (d)] at $15 \mathrm{~K}$ with different injection current. The inset shows the NPB-mixed devices structure (front panel) and schematic of interaction between charge carriers and triplets in the exciton formation zone (bottom panel).

dependence of MEL in two devices. Under the same injection current of $200 \mu \mathrm{A}$, the high field part of MEL in unmixed-device (Fig. 1(c)) exhibits more remarkable decay when the temperature comes to low, while less temperature dependence in the NPB-mixed device (Fig. 1(d)).

These observations indicate that the introduction of NPB into the $\mathrm{Alq}_{3}$ EML results in the change of MEL. For the enlargement of the low field effect, there are two possible explanations: (1) the larger electron-hole separation distant due to the insertion of NPB molecule between $\mathrm{Alq}_{3}$ molecules, causing a smaller singlet-triplet exchange energy and more effective conversion between singlet and triplet excitons through hyperfine interaction; ${ }^{12}$ (2) the reduced charge mobility in the NPB-mixed EML. ${ }^{13}$ For the weakened high field decay of MEL upon NPB mixing, as the main point in the following discussion, we suggest a suppressed TTA interaction in the NPB-mixed devices, which may result from the reduction of triplet exciton density.

Before establishing the relationship between the high field decay of MEL and the triplet exciton density, it is essential to rule out the influence of magneto-conductance $(\mathrm{MC}=\Delta \mathrm{I} / \mathrm{I}=(I(\mathrm{~B})-(0)) / I(0))$ change $^{11}$ upon NPB mixing. Figs. 2(a) and 2(b), respectively, show the MC of OLEDs without and with the NPB-mixed EML at $15 \mathrm{~K}$. Similar to the trace of MC of the unmixed-device, all the MC curves of NPB-mixed device exhibit a rapid rising and then saturated trends. No high field decay of MC upon the NPB mixing

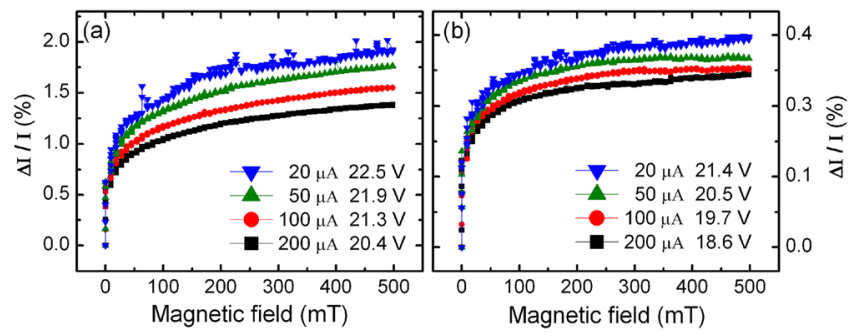

FIG. 2. (Color online) $\Delta \mathrm{I} / \mathrm{I}$ versus magnetic field (MC) for (a) unmixed devices and (b) $50 \%$ NPB-mixed devices at $15 \mathrm{~K}$ with different injection current from 20 to $200 \mu \mathrm{A}$. indicates that the MC change is not the cause for the MEL change in the NPB-mixed device.

In order to quantify the triplet exciton density in the NPB-mixed devices, we conduct a simple calculation based on the rate model. ${ }^{16,17}$ When the TTA interaction is considered in fluorescent OLEDs, the EL intensity should include two parts: the prompt fluorescence $(\mathrm{PF})$ from direct charge combination and delayed fluorescence (DF) from the TTA interaction, i.e., $E L=E L_{\mathrm{PF}}+E L_{\mathrm{DF}}$. Therefore, the MEL can be expressed as ${ }^{17}$

$$
\mathrm{MEL}=\Delta \mathrm{EL} / \mathrm{EL}=\frac{\mathrm{m}}{\mathrm{m}+1} \frac{\Delta E L_{P F}}{E L_{P F}}+\frac{1}{\mathrm{~m}+1} \frac{\Delta E L_{D F}}{E L_{D F}},
$$

where $\mathrm{m}=E L_{\mathrm{PF}}(0) / E L_{\mathrm{DF}}(0)$, denoting the ratio of PF to $\mathrm{DF}$ without magnetic fields.

By using the values of MEL at $125 \mathrm{mT}$ and $200 \mathrm{mT}$ from experimental curve, we can get the value of the DF fraction from TTA interaction named $\beta .^{17}$ Because the output of TTA interaction is proportional to the square of the triplet exciton density $\beta=\frac{\mathrm{k}_{\mathrm{TT}}}{\sqrt{2 \beta}}[T]^{2},{ }^{18}$ the triplet exciton density [T] is estimated by $\sqrt{\frac{2 \beta}{k_{T T}}}$, where $\mathrm{k}_{\mathrm{TT}}$ is the interaction constant of TTA.

So, we can calculate the triplet exciton density in the NPB-mixed devices with different NPB concentrations based on the experimental curves, as shown in the right coordinate of Fig. 3(a). From the experimental MEL curves of NPB-mixed devices (Fig. 3(b)), we also figure out the high fields effect of MEL defined as $\mathrm{HFE}=\left.\mathrm{MEL}\right|_{\mathrm{B}}=45 \mathrm{mT}$ $-\left.\operatorname{MEL}\right|_{\mathrm{B}=500 \mathrm{mT}}$ (denoting the high fields decay of MEL), ${ }^{11}$ synchronously displayed in the left coordinate of Fig. 3(a) in order to examine the relationship between the HFE and the triplet exciton density. Interestingly, they show quite similar NPB concentration dependence: (1) When EML is mixed with NPB below 50 wt. \%, they both gradually decrease and reach to the minimum (2) By further increasing the NPB ratio over $50 \mathrm{wt}$. \%, their magnitudes recover and increase close to the value of unmixed case. The similar trend of MEL and triplet exciton density versus NPB concentration reveals a possible method to tune the MEL through adjusting the triplet exciton density in OLEDs. In the following discussion, we will illustrate how the mixing of NPB into $\mathrm{Alq}_{3}$ EML varies the number of triplet excitons.

As compared with the static charges accumulating on the $\mathrm{NPB} / \mathrm{Alq}_{3}$ interface (space charges), the moving charges have higher efficiency in quenching the triplet excitons because the interaction between charge and triplet excitons is a charge velocity-determined reaction. ${ }^{4,15}$ So, if the space

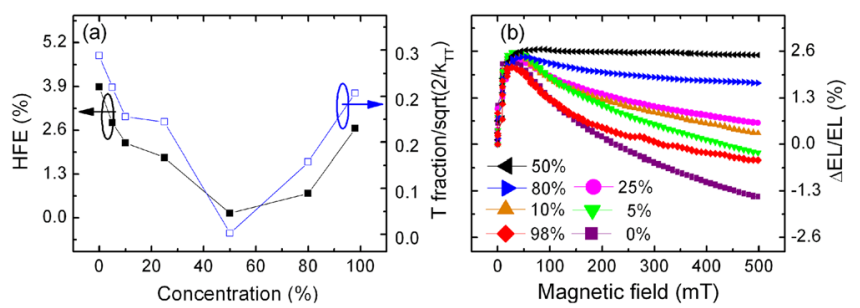

FIG. 3. (Color online) At $15 \mathrm{~K}$, (a) NPB concentration dependence of MEL high field decay and triplet excitons density and (b) MEL for NPB-mixed devices with different concentrations. 


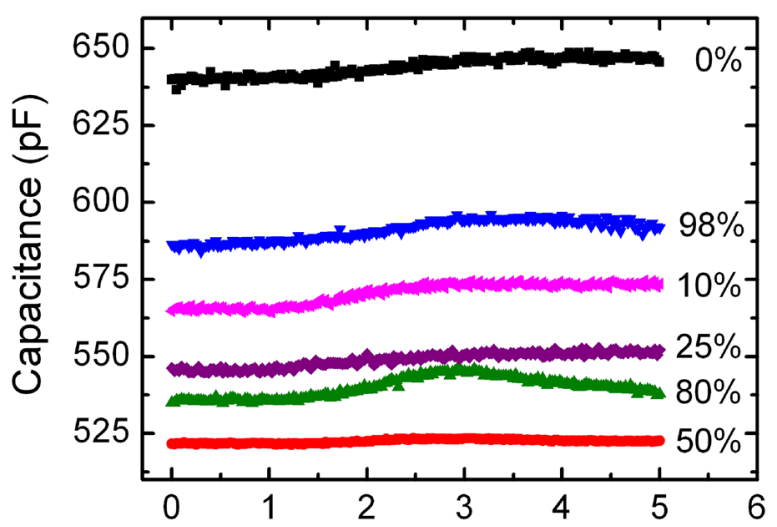

FIG. 4. (Color online) The capacitance-voltage characteristics of the NPBmixed devices with voltage bias ranging from 0 to $5 \mathrm{~V}$.

charges can be released into the free charges, the triplet exciton density will be reduced considerably. By mixing NPB molecular into the pristine $\mathrm{Alq}_{3} \mathrm{EML}$, the abrupt interface between NPB layer and $\mathrm{Alq}_{3}$ layer will be destroyed. ${ }^{19}$ Thus, under the external electric fields, without forming the space charges accumulating at the NPB/Alq 3 interface, the injected holes and electrons can drift through the NPB-mixed EML (exciton formation zone) and then effectively interact with the triplet excitons (as depicted in the inset of Fig. 1(d)). What is more, the NPB molecules can be regarded as scatter centers for the mobile electrons collision and detour ${ }^{20}$ and, consequently, increasing their probability to interact with triplet excitons owing to their increased transit path and residence time in the exciton formation zone. Another factor may contribute to the reduction of triplet excitons is that: the reduced recombination rate due to the penetration of charge carriers into the EML, ${ }^{19}$ reflected by relatively lower quantum efficiency as compared with the unmixed case (data not shown here). By considering these two factors, we can expect the reduced triplet excitons density due to the interaction with moving charge carriers.

Indeed, this assumption can be reflected from Fig. 3(a) where the calculated triplet exciton density initially decreases with increasing NPB concentration up to $50 \mathrm{wt}$. \%. Although the following increase of triplet exciton density over $50 \mathrm{wt}$. \% may appear to be anomalous with the above analysis, we should note that the mixed EML with very high NPB concentration (hence very low concentration of $\mathrm{Alq}_{3}$ ) will behave primarily as a hole transport layer with fewer charge carriers flowing through the exciton formation zone. Therefore, the charge-triplets interaction is weakened and triplet exciton density recovers.

Since the device capacitance is a useful tool to implicate the interfacial states of space charges, ${ }^{21}$ we conduct $\mathrm{C}-\mathrm{V}$ tests of NPB-mixed devices to check whether the NPBmixing can eliminate the space charges. As shown in Fig. 4, the capacitances of the NPB-mixed devices are lower than that of the unmixed device, suggesting the remarkable reduction of the space charges accumulating at the $\mathrm{NPB} / \mathrm{Alq}_{3}$ interface. This means that under the same voltage bias, the injected charges will more easily flow into the exciton formation zone without forming the space charges, leading to a significant triplet excitons quenching. In addition, we also see that the device capacitance first decrease with the NPB concentration until 50 wt. \%, and then recover. This trend provides another evidence for the population change of charge carriers flowing through the exciton formation zone.

In summary, we have demonstrated a method to tune the MEL in $\mathrm{Alq}_{3}$-based OLEDs by mixing the hole transport material NPB into the $\mathrm{Alq}_{3}$ EML. Theoretical and experimental results exhibit a consistent variation of MEL and triplet excitons with NPB concentration, revealing a possible way to tune the MEL by adjusting the density of triplet excitons. The interaction between triplet excitons and charge carriers flowing through the exciton formation zone was employed to explain the change of triplet excitons density, and then the change of MEL. The variation of capacitance of the NPB-mixed devices gives further verification of the elimination of space charges.

This work was supported by the NSF of Chongqing (CSTC, 2010BA6002), the National Natural Science Foundation (NNSF) of China (Grant Nos. 10974157 and 10974159), and the Grants (Nos. HKU712010E and HKU712108) from the Research Grants Council of the HK Special Administrative Region, China.

${ }^{1}$ F. J. Wang, H. Bassler, and Z. V. Vardeny, Phys. Rev. Lett. 101, 236805 (2008).

${ }^{2}$ J. Kalinowski, M. Cocchi, D. Virgili, P. Di Macro, and V. Fattori, Chem. Phys. Lett. 380, 710 (2003).

${ }^{3}$ P. Desai, P. Shakya, T. Kreouzis, and W. P. Gillin, Phys. Rev. B. 75, 094423 (2007).

${ }^{4}$ B. Hu, L. Yan, and M. Shao, Adv. Mater. 21, 1500 (2009).

${ }^{5}$ G. Veeraraghavan, T. D. Nguyen, Y. Sheng, O. Mermer, and M. Wohlgenannt, J. Phys.: Condens. Matter. 19, 0362 (2007).

${ }^{6}$ F. L. Bloom, M. Kemerink, W. Wagemans, and B. Koopmans, Phys. Rev. Lett. 103, 066601 (2009).

${ }^{7}$ A. H. Davis and K. Bussmann, J. Vac. Sci. Technol. A. 22, 1885 (2004).

${ }^{8}$ C. Gärditz, A. G. Mückl, and M. Cölle, J. Appl. Phys. 98, 104507 (2005).

${ }^{9}$ Y. Zhang, R. Liu, Y. L. Lei, and Z. H. Xiong, Appl. Phys. Lett. 94, 083307 (2009).

${ }^{10}$ T. D. Nguyen, G. Hukic-Markosian, F. J. Wang, L. Wojcik, X. G. Li, E. Ehrenfreund, and Z. V. Vardeny, Nature Mater. 9, 345 (2010).

${ }^{11}$ P. Chen, Y. L. Lei, Q. L. Song, Y. Zhang, R. Liu, Q. M. Zhang, and Z. H. Xiong, Appl. Phys. Lett. 95, 213304 (2009).

${ }^{12}$ H. Odaka, H. Okamoto, M. Kawasaki, and Y. Tokura, Appl. Phys. Lett. 88, 123501 (2006).

${ }^{13}$ B. F. Ding, Y. Yao, Z. Y. Sun, C. Q. Wu, X. D. Gao, Z. J. Wang, X. M. Ding, W. C. H. Choy, and X. Y. Hou, Appl. Phys. Lett. 97, 163302 (2010).

${ }^{14}$ P. A. Bobbert, T. D. Nguyen, F. W. A. van Oost, B. Koopmans, and M. Wohlgenannt, Phys. Rev. Lett. 99, 216801 (2007).

${ }^{15}$ B. Hu and Y. Wu, Nature Mater. 6, 985 (2007).

${ }^{16}$ Y. Iwasaki, T. Osasa, M. Asahi, M. Matsumura, Y. Sakaguchi, and T. Suzuki, Phys. Rev. B. 74, 195209 (2006).

${ }^{17}$ Y. L. Lei, Q. L. Song, P. Chen, F. Li, Q. M. Zhang, Y. Zhang, and Z. H. Xiong, Org. Electron. 12, 1512 (2011).

${ }^{18}$ R. E. Merrifield, J. Chem. Phys. 48, 4318 (1968).

${ }^{19}$ Y. C. Luo and H. Aziz, Appl. Phys. Lett. 95, 073304 (2009).

${ }^{20}$ S. C. Tse, K. C. Kwok, and S. K. So, Appl. Phys. Lett. 89, 262102 (2006).

${ }^{21}$ G. Garcia-Belmonte, H. J. Bolink, and J. Bisquert, Phys. Rev. B. 75, 085316 (2007). 